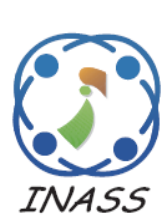

\title{
Q-Learning Hybrid Type-2 Fuzzy Logic Control Approach for Photovoltaic Maximum Power Point Tracking Under Varying Solar Irradiation Exposure
}

\author{
Reza Fauzi Iskandar ${ }^{1,4} \quad$ Edi Leksono $^{2 *} \quad$ Endra Joelianto $^{3}$ \\ ${ }^{1}$ Department of Engineering Physics, Faculty of Industrial Technology, Institut Teknologi Bandung, Indonesia \\ ${ }^{2}$ Engineering Physics Research Group, Faculty of Industrial Technology, Institut Teknologi Bandung, Indonesia \\ ${ }^{3}$ Instrumentation and Control Research Group, Faculty of Industrial Technology, Institut Teknologi Bandung, Indonesia \\ ${ }^{4}$ Engineering Physics, School of Electrical Engineering, Telkom University, Indonesia \\ *Corresponding author's Email: edi@tf.itb.ac.id
}

\begin{abstract}
This paper presents a control strategy of maximum power point tracking and focuses on hybrid q-learning and type-2 fuzzy logic control strategy. Photovoltaics have non-linear voltage and current characteristics which influenced by temperature and exposure of solar irradiation so that the maximum power point (MPP) can change at any time. Unfortunately, operating conditions outside the MPP could reduce the efficiency of electrical power transfer from the photovoltaic system to the load. Q-learning (QL) has the potential to provide action decisions of duty cycle signal based on the state of the power gradient percentage range. The Q-learning hybrid method with Type-2 fuzzy logic control is proposed to provide correction of QL control signal by considering the uncertainty direction of power point shifting. The proposed strategy is important particularly to avoid a sudden change in solar irradiation exposure. The simulation results show that the Q-learning hybrid type-2 fuzzy logic control based MPPT response in the photovoltaic control system has tracking efficiency about $97 \%$, rapid rise time of $0.08 \mathrm{~s}$, settling time of $0.23 \mathrm{~s}$, low overshoot and stable response for handling the change of irradiation and temperature exposure simultaneously.
\end{abstract}

Keywords: Maximum power point tracking, Photovoltaic system, Q-learning, Type-2 fuzzy logic.

\section{Introduction}

Photovoltaic system is one of the fastest growing renewable energy conversion and technologies, the energy source is very abundant, friendly to the environment, noiseless, clean and has adjustment flexibility to the required electricity production capacity. Photovoltaic system is proven for largescale to small-scale electricity such as district electricity, residence, households, and street lighting. Research and innovation in increasing the efficiency and optimization of electric generation are the key to support reliable and sustainable energy infrastructure [1].

The development of maximum power points tracking (MPPT) method is one effort to increase the efficiency of photovoltaic systems. The MPP method has been developed from traditional to artificial intelligence (AI). The motivation for the development of the AI method in this area is mainly driven by the issue of global MPP deal with nonlinear systems and changes in environmental conditions such as solar irradiation shifting and ambient temperature [2]. Photovoltaics have nonlinear voltage and current characteristics which influenced by temperature and exposure of solar irradiation so that the maximum power point (MPP) can change at any time. Operating conditions outside the MPP could reduce the efficiency of electrical power transfer from the photovoltaic system to the load [3]. Consequently, the artificial intelligent-based MPPT is important to be considered in order to maintain the MPP.

Several artificial intelligent-based MPPT methods explained in the literature [1] have been used widely and successfully such as fuzzy logic control (FLC), artificial neural network (ANN), sliding mode control (SMC), and particle swarm optimization (PSO). PSO has been investigated in [4], the system 
performance achieves high power tracking efficiency about $99.98 \%$, however, unfortunately, it has not been avoided from the power response oscillation problem. Several mixed methods with SMC have been reported. The researcher interested with SMC due to robustness and rapid response. Hybrid strategy of PSO-SMC [5] meets high accuracy and fast transient response yet cannot dispel of power undershoot response. Some other mixed method such as ANN-Backstepping SMC and high gain observer [6], SMC and high gain observer [7], backstepping integral sliding mode controller (BISMC) [8] have been developed by researcher with accurate and fast response.

FLC [9] performs fast-tracking and low steadystate fluctuation characteristic. However, varying of solar irradiation conditions will cause oscillation, drift, and uncertainty problems in MPP tracking. Fuzzy neural network (FNN) [10] yield power tracking efficiency about $96 \%$, however still suffers from overshoot when temperature changes occur. The MPP achievement can be observed from the P-V curve where its gradient close to zero. Moreover, it can be approached by step-up or step-down of the voltage converter, while the rate needs to adjust, and the uncertainty problem of the power point shifting must be considered to achieve a more precise result.

Type-2 fuzzy logic (T2FL) allows designer to accommodate the uncertainty of the gradient and shift direction of power points in the upper and lower boundary of membership function (MF) ranges, this is clearly different from type-1 fuzzy logic (T1FL) which tends so tied to MF absolute value. Comparative study of T1FL and T2FL in temperature control problem have been observed in [11] which T2FL perform successful results. The ability of T2FL was studied in determining more precise of medical diagnostic decisions [12].

Reinforcement learning (RL) has become attention in recent years and surprising of conquering most challenge in difficult game [13], machine would be more active than smarter one, considering strategic options, efficiency and data need [14]. RL is a significant method, especially in dealing with optimization problems through a learning strategy in the process of interaction with the environment. In Qlearning, as a type of RL, the results of the learning process will be accumulated in a table of quality. The optimum condition will be indicated by the achievement of the highest reward and quality. This table would be interested to accelerate finding the optimum point for each tracking condition.

The main contribution of this paper is aimed to investigate the performance of hybrid expert Qlearning and type-2 fuzzy logic controller as part of intelligent control in MPP tracking problem of photovoltaic systems. Q-learning has the potential to provide action decisions of duty cycle signal based on the state of the power gradient percentage range. Meanwhile, T2FL logic aim to provide a duty cycle correction signal based on the power gradient and direction of track point shifting by considering the range of uncertainty. Moreover, according to result of proposed method, it is interesting as compared to classical T1FL control with various of membership function forms and QL.

This paper is constructed as follows, the second section presents the modelling of photovoltaic systems and maximum power point descriptions. The third section presents the control strategy, this section also describes the potential Q-learning that provides the expert table of optimum duty ratio and type- 2 fuzzy logic which dedicated for voltage uncertainty regulation. Q-learning is expected to provide the rapid optimum duty ratio, consequently the hybrid type-2 fuzzy logic and Q-learning is addressed to provide improvement of transient and stable response. The simulation result and discussion have been explained in section 4, which are resumed by the conclusion in the last section.

\section{System description and mathematical modelling}

The structure of the considered photovoltaic system in this paper is shown in Figure. 1 which consists of a solar power plant prototype based photovoltaic, buck-boost converter and resistive load. Photovoltaic energy conversion performance is influenced by the intrinsic non-linear characteristics, solar irradiation, temperature, and load conditions. This condition makes it difficult for the system to reach its maximum power point. The buck-boost converter is controlled by a standard PWM signal and used as an actuator for active tracking of maximum power points thereby increasing the operating efficiency of the photovoltaic system. The mathematical model of the system is described in the section below.

\subsection{Photovoltaic and maximum power point}

Photovoltaic is a semiconductor that has conduction and valence bands. Solar irradiation exposure on photovoltaic surfaces, which an amount of photon energy equal to or greater than the energy gap causes electrons in the weak valence band to move into the conduction band, becomes electric currents in the circuit. Figure. 2 is a photovoltaic cell model [15] which has been equated with an 


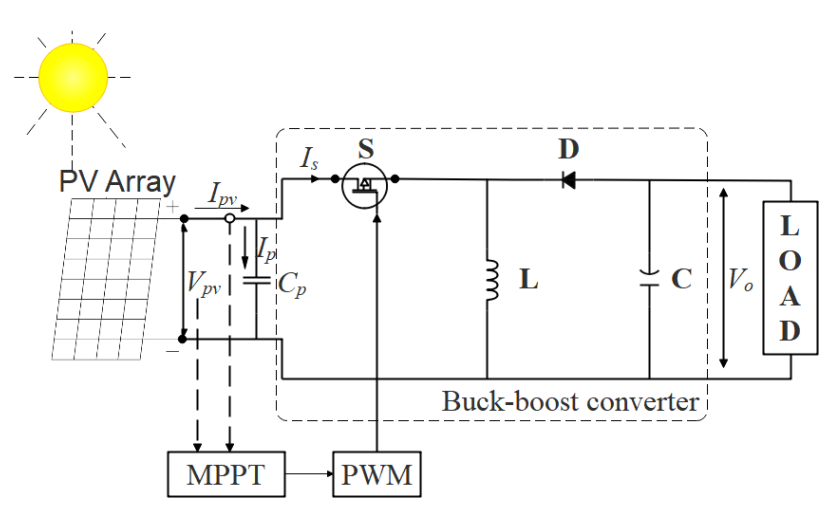

Figure. 1 Structure of considered photovoltaic system

equivalent electrical circuit consist of a current source, diode, resistors connected in series and in parallel circuit. $I_{p h}$ is the photocurrent generated from the photovoltaic conversion process, $R_{s h}$ is the shunt resistance which represents the leakage current at the cell boundary, and $R_{s}$ is the series resistance that comes from the intrinsic resistance of photovoltaic material.

Applying Kirchoff's current law to photovoltaic equivalent circuit in Figure. 2 yields current $I=$ $I_{p h}-I_{d}-I_{p}$ where diode current $I_{d}$ and parallel current $I_{p}$ across to shunt resistor presented by Eq. (1) and (2) respectively.

$$
\begin{gathered}
I_{d}=I_{0}\left[\exp \left(\frac{V+I \cdot R_{s}}{a}\right)-1\right] \\
I_{p}=\frac{V+I \cdot R_{s}}{R_{s h}} \\
a=\frac{N_{s} \cdot A \cdot k \cdot T_{c}}{q}=N_{s} \cdot A \cdot V_{T}
\end{gathered}
$$

where $a, k, T_{c}, q, N_{s}, A$ are thermal voltage, Boltzman constant, photovoltaic temperature, electron charge, number of serial photovoltaic, and ideality factor, respectively. According to Eq. (1) and (2), the photovoltaic current equation can rewritten as shown in Eq. (4).

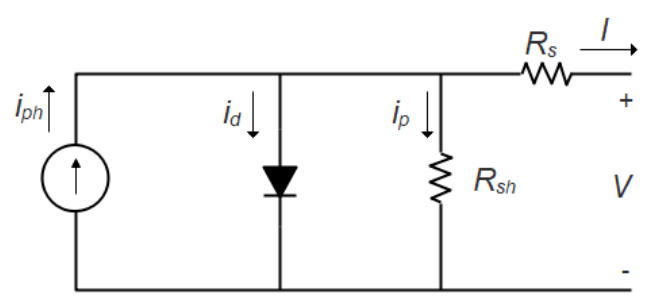

Figure. 2 Equivalent circuit of photovoltaic cell

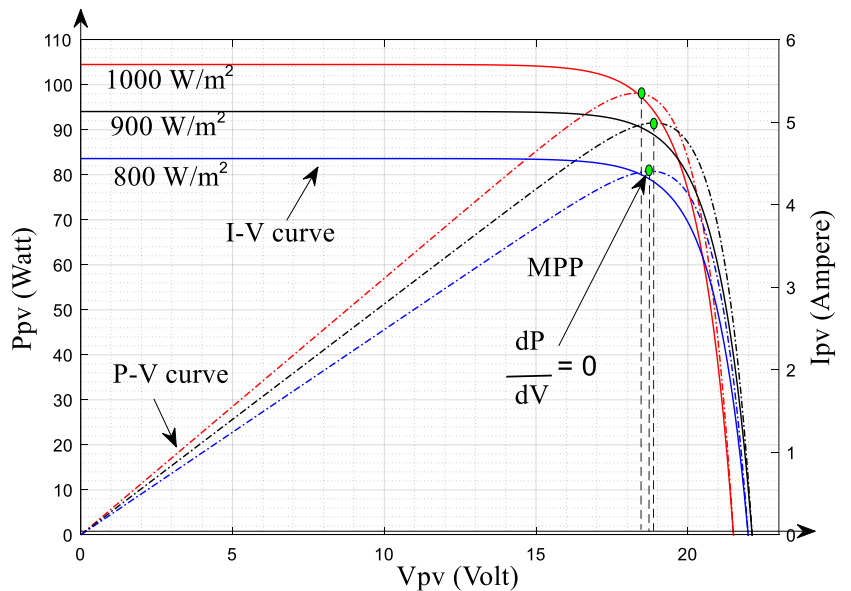

Figure. 3 Characteristic of photovoltaic in different irradiance level

$I=I_{p h}-I_{0}\left[\exp \left(\frac{V+I \cdot R_{s}}{a}\right)-1\right]-\frac{V+I \cdot R_{s}}{R_{s h}}$

The power to voltage curve of photovoltaic can be used as one basis for maximum power point estimation. The P-V curve variation of photovoltaic in different solar irradiation and temperature are shown in Figure. 3. According to this figure, MPP value for each condition $\left(800 \mathrm{~W} / \mathrm{m}^{2}, 25^{\circ} \mathrm{C}\right),(900$ $\left.\mathrm{W} / \mathrm{m}^{2}, 27^{\circ} \mathrm{C}\right)$ and $\left(1000 \mathrm{~W} / \mathrm{m}^{2}, 32^{\circ} \mathrm{C}\right)$ are around 82.15 W, 91.5 W and $98.1 \mathrm{~W}$, respectively.

\subsection{Buck-boost converter}

Buck-boost converter is a type of $\mathrm{dc} / \mathrm{dc}$ converter that produces a voltage magnitude greater or less than the input voltage through a switching regulation mechanism. Figure. 4 shows the basic circuit of converter [16]. MOSFET based element present for substitute switching device as indicated in Figure. 1 The standard PWM signal from the control element may employ to adjust the MOSFET saturation or cut-off state, so that the output voltage of the $\mathrm{dc} / \mathrm{dc}$ converter can be modified. The converter is designed for $40 \mathrm{kHz}$ switching frequency, minimum resistive load about $1 \mathrm{Ohm}$ and duty cycle range $0-0.8$.

When the MOSFET is saturated or switch-on and the diode is reverse biased then electrical energy will be stored to inductor with a voltage value $V_{L}$

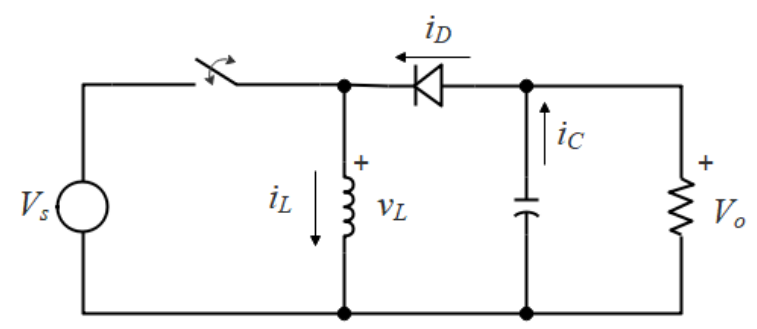

Figure. 4 Buck-boost converter 
which equal to source voltage $V_{S}$.

$$
V_{L}=V_{S}=L \frac{d i_{L}}{d t}
$$

since the change in current is relatively constant, Eq. (5) can be rewritten in Eq. (6)

$$
\frac{\Delta i_{L}}{\Delta t}=\frac{\Delta i_{L}}{D T}=\frac{V_{s}}{L}
$$

based on Eq. (1), the current in the inductor when switched-on is

$$
\left(\Delta i_{L}\right)_{\text {closed }}=D T \frac{V_{S}}{L}
$$

which $D, T$ and $L$ were a duty ratio, time interval, and inductance, respectively. The condition when the MOSFET is being cut-off or switch-off state and the diode is forward bias, therefore current flows towards the resistor and capacitor so that the inductor voltage $V_{L}$ value was equal to output voltage $V_{o}$

$$
\frac{\Delta i_{L}}{\Delta t}=\frac{\Delta i_{L}}{(1-D) T}=\frac{V_{o}}{L}
$$

so that the current change in inductor when switchoff moment presented in Eq. (9)

$$
\left(\Delta i_{L}\right)_{\text {open }}=(1-D) T \frac{V_{o}}{L}
$$

Consider Eq. $(7,9)$ and the MOSFET being switchoff, then the relation between input and output voltage is shown in Eq. (10)

$$
V_{o}=-\frac{D}{(1-D)} V_{S}
$$

The buck-boost converter is a combination of buck and boost modes so the output voltage can be adjusted to be smaller or greater than the input. From Eq. (10) conclude that voltage profile can be modified by changing the duty ratio $D$, where $D>0.5$ produces a step-up voltage while $D<0.5$ produces a step-down. The shifting direction of $D$ value is determined by condition of power gradient against voltage, and the value become zero while the MPP position was reached. This simulation applies the power electronic diode which implements a diode in parallel with a series RC snubber circuit to reduce a spike and losses due to switching. The value of resistance $R_{o n}$, forward voltage $V_{f}$, snubber resistance
$\mathrm{R}_{\mathrm{s}}$, and snubber capacitance $\mathrm{C}_{\mathrm{s}}$ are $0.1 \mathrm{~m} \Omega$, 0Volt, $500 \Omega$ and $0.25 \mathrm{nF}$, respectively.

\section{Control system}

The proposed control system is expressed in diagram block Fig. (6) The main objective of this control scheme is to investigate the effect of Qlearning (QL) hybrid type-2 fuzzy logic (T2FL) control in regulating the power of the photovoltaic system in order to guarantee system operation at its maximum power point. QL has been designed to generate immediate recommendations for optimal duty-cycle change values based on power gradient information $d P / d V$ or $m$. Furthermore, T2FL serves to provide a correction signal based on the power gradient information $m$ and gradient changes $\Delta m$, moreover, T2FL aim to accommodate uncertainty of $m$ and $\Delta m$ which may arise from change in solar irradiation and temperature.

QL and T2FL control signals result, namely QLT2FL, follow the Eq. (5) which is the signal that determines the value of step-up or step-down mode in buck-boost converter. The duty cycle threshold is defined by 0.5 , so the condition QLT2FL > 0 causes the duty-cycle value move up and the converter performs a voltage step-up, and vice versa.

$$
\delta_{Q L T 2 T L}=\Delta D_{\text {Threshold }} \pm \Delta D_{Q L} \pm \Delta D_{T 2 F L}
$$

\subsection{Q-learning design}

The reinforcement learning (RL) method is one of the studies in machine learning that works based on a cumulative reward of an agent from the environment in making the right action decisions to achieve the goals [5]. In this study, Q-learning (QL) which is part of RL, is used. Figure. 7 is the basic QL structure which composed by several main functions such as action, state, reward, environment, and table of quality [17].

QL will map the state $S_{n}$ and action $A_{n}$ to a table called Q-table. The table initially set by zero, through

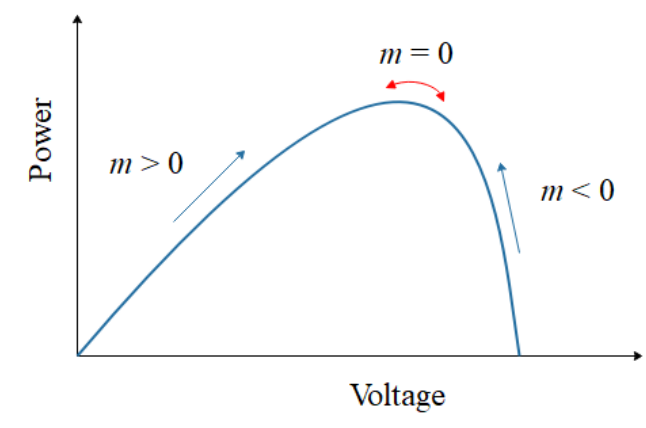

Figure. 5 Power gradient of P-V curve 


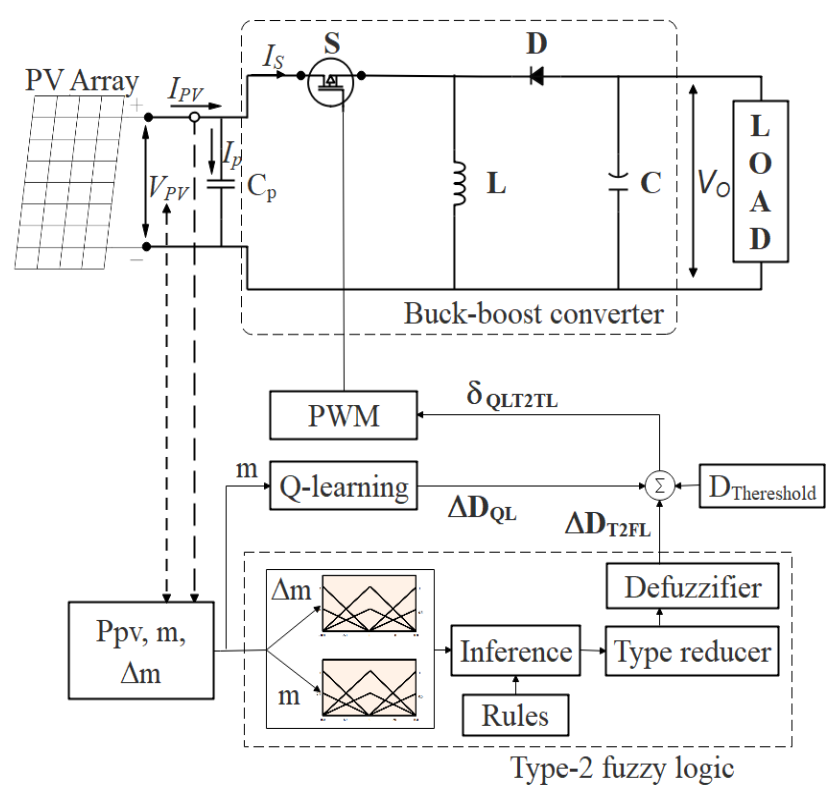

Figure. 6 Block diagram of proposed control system

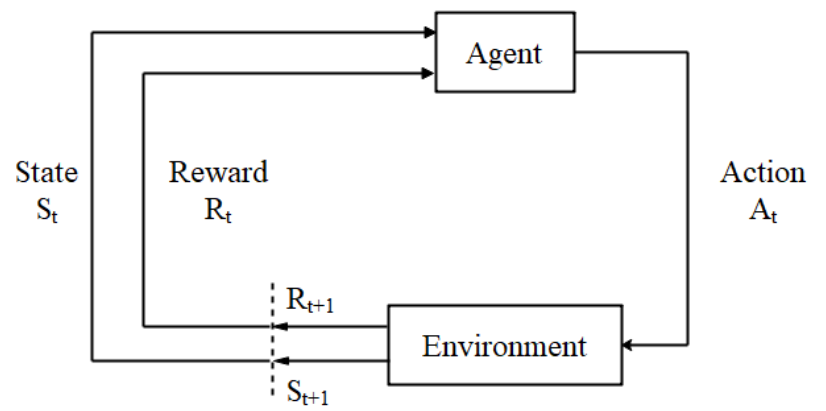

Figure. 7 Q-learning structure

an iterative process will be updated by considering various possible actions that can be taken and the consequences of the prize $r$ will be received. The process was calculated using the Bellman Eq. (12)

$$
\begin{aligned}
Q^{\prime}(s, a)=Q(s, a) & \\
& +\alpha\left[r+\gamma \cdot \max Q\left(s^{\prime}, a^{\prime}\right)\right. \\
& -Q(s, a)]
\end{aligned}
$$

where $Q^{\prime}, Q, \alpha, s^{\prime}, a^{\prime}$ and $\gamma$ are quality table update, past quality table, learning rate, next state, next action, and discount factor, respectively. Figure. 8 is an iterative flowchart of Q-learning to update Qtable. The state variable is power gradient point $m$ in $\mathrm{PV}$ curve and the action is duty-ratio change $\Delta D$ as shown in

Table 1.

This paper presents five state $S_{n}$ for power point gradient condition $[0-0.5 \%, 2.5-5 \%, 5-10 \%, 10-50 \%$, 50-100\%] and five action $A_{n}$ represents the possible applicable $\Delta \mathrm{D}_{\mathrm{QL}}$ value $[ \pm 0.02, \pm 0.15, \pm 0.01, \pm 0.005$,
$0]$. The reward table referred to the highest point will be given to the nearest condition from MPP, and vice versa. The Q-table is updated for each episode such

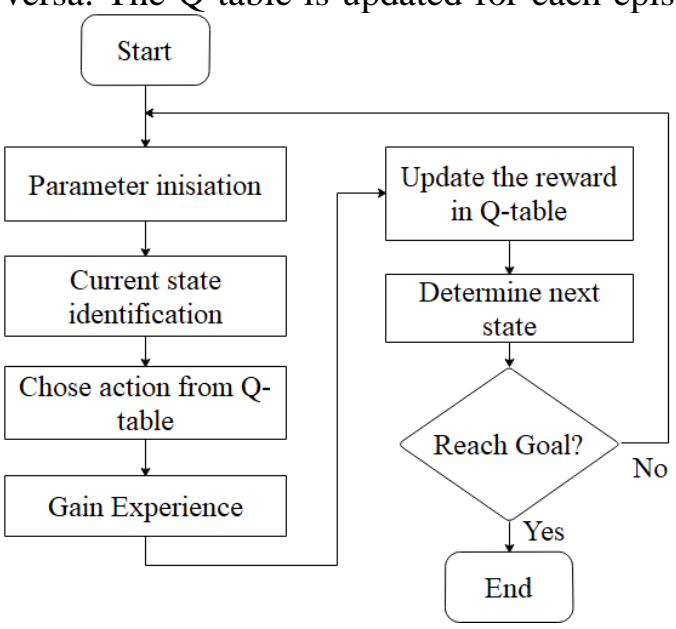

Figure. 8 Q-learning flowchart

\begin{tabular}{|c|}
\hline Algorithm 1 Q-learning [18] \\
\hline Initialize $\theta, \alpha, \gamma$ \\
For each episode : \\
set initial $\mathrm{k}=0$, initialize $x_{0}$ \\
Until $x_{k+1}$ is terminal : \\
choose $u^{(j)} \in U$ using $\pi_{\theta}$ \\
perform $u_{k}=u^{(j)}$ \\
observe state $x_{k+1}$ and reward $r\left(x_{k}, u_{k}\right)$ \\
$i \leftarrow \arg \max \theta_{l}^{T} \varphi\left(x_{k+1}\right)$ \\
$\delta \leftarrow r\left(x_{k}, u_{k}\right)+\gamma \theta_{i}^{T} \varphi\left(x_{k+1}\right)-\theta_{j}^{T} \varphi\left(x_{k}\right)$ \\
$\theta \leftarrow \theta+\alpha \delta \varphi\left(x_{k}\right)$ \\
$x_{k} \leftarrow x_{k+1}$ \\
update $k \leftarrow k+1$
\end{tabular}

that achieved the condition where the gradient of PV curve equal to zero, which means the MPP point has been reached. Q-learning program was indicated in Algorithm 1.

The convergence Q-table would be used for expert map selection for driving the control action. The best action according to QL is a Q-table column which has a maximum value in particular row corresponding to related state. The action is selected and immediately applied as control signal and continues until reaches the maximum power point.

\subsection{Type-2 Fuzzy logic control design}

The main purpose of T2FL is to deal with information uncertainty in order to improve system performance [19]. The probable value in membership function (MF) of T2FL for each corresponding input value is not absolute but a range values between the upper and lower limits as shown in Fig (9) [20].

In this paper, the MF form is addressed for $m$ and $\Delta m$ with an equal input range [-100 100] in order to 
anticipate a drastic change that may occur by change in solar irradiation and ambient temperature.

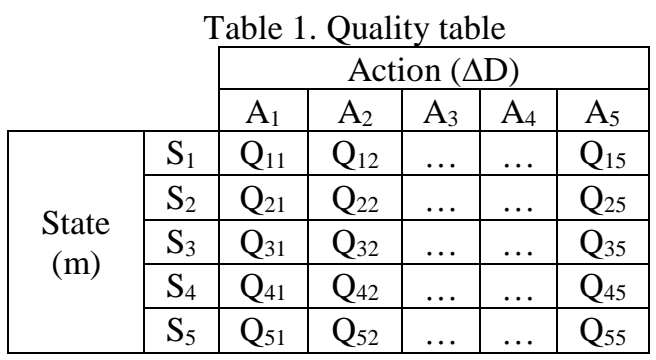

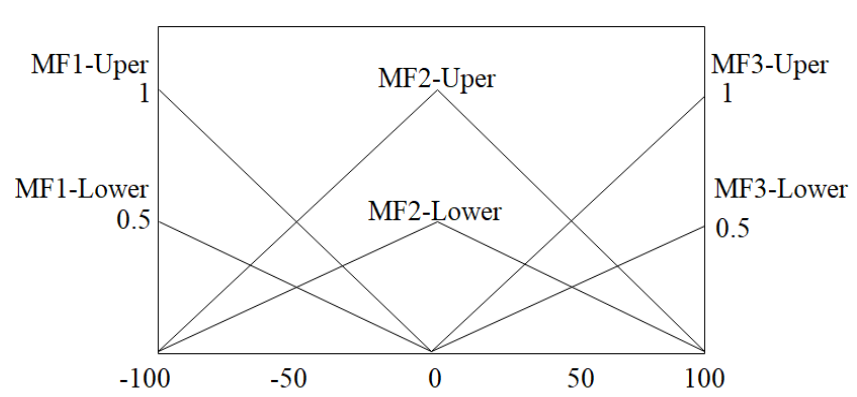

Figure. 9 Membership function for $m$ and $\Delta m$

\section{Simulation result and discussion}

This section shows performance results. The proposed QLT2FL control will be evaluated in MATLAB/SIMULINK simulation and then compared to QL, T1FL-triangle, T1FL-trapezoid, T1FL-gaussian performances and well established FNN. The control of photovoltaic system was simulated with solar irradiation input condition and temperature change every 0.5-time interval; $800 \mathrm{~W} / \mathrm{m}^{2}, 1000 \mathrm{~W} / \mathrm{m}^{2}$, and $900 \mathrm{~W} / \mathrm{m}^{2}$ as shown in upper of Figure. 15 (a).

The Fig. 10 shows the surface graph of Q-table which is the evolution of the power gradient related to the corresponding duty ratio. The Q-table has dimensions of $5 \times 5$ and consists of 25 matrix elements.

Table 2. Characteristic of SMP100-M module

\begin{tabular}{|l|c|}
\hline \multicolumn{2}{|c|}{$\begin{array}{c}\text { Electrical characteristic } \\
\text { value at standard test condition STC } \\
\left.\text { (AM } 1.5,1000 \mathrm{~W} / \mathrm{m}^{2}, 25^{\circ} \mathrm{C}\right)\end{array}$} \\
\hline Max power $P_{\max }$ & $100 \mathrm{Wp}$ \\
\hline Max power voltage $V_{m p}$ & $18.75 \mathrm{~V}$ \\
\hline Max power current $I_{m p}$ & $5.35 \mathrm{~A}$ \\
\hline Open circuit voltage $V_{o c}$ & $22.53 \mathrm{~V}$ \\
\hline Cell efficiency & $17 \%$ \\
\hline Modul efficiency & $15.10 \%$ \\
\hline
\end{tabular}

Table 3. Buck-boost converter specification

\begin{tabular}{|l|c|}
\hline Switching Frequency $(f)$ & $40 \mathrm{KHz}$ \\
\hline Inductor & $125 \mu \mathrm{H}$ \\
\hline Output capacitor & $2200 \mu \mathrm{F}$ \\
\hline
\end{tabular}

\begin{tabular}{|l|c|}
\hline Input capacitor & $350 \mu \mathrm{F}$ \\
\hline Minimum load & $1 \Omega$ \\
\hline Duty-ratio & $0-0.8$ \\
\hline
\end{tabular}

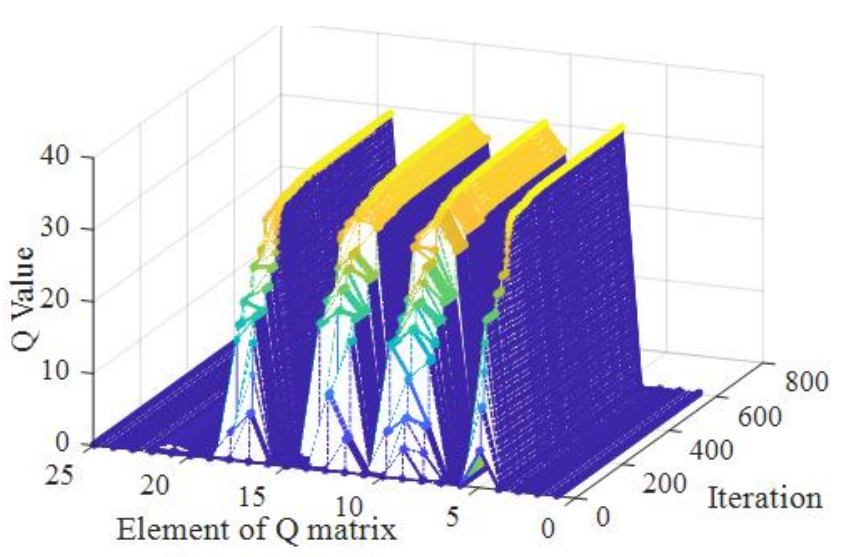

Figure. 10 Surface of Q-table

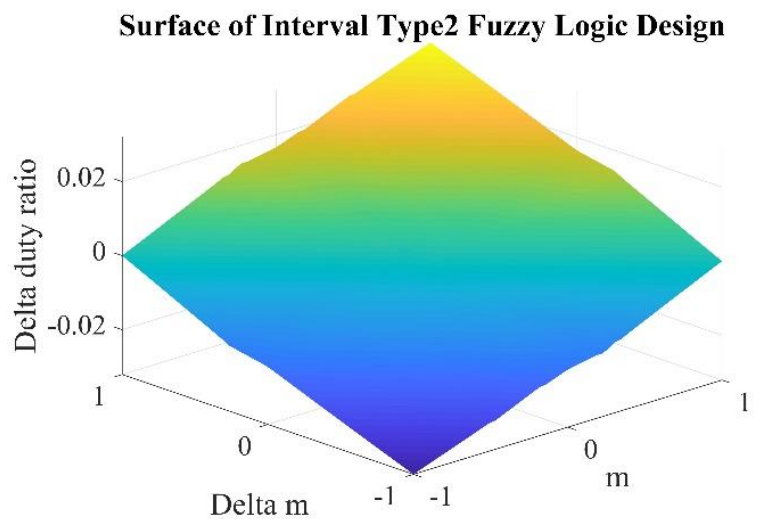

Figure. 11 Surface of type-2 fuzzy logic

According to the figure, it can be seen the evolutionary convergence of each element in the Qtable that has been trained in 100 episodes. Meanwhile, Fig. 11 shows the surface of type-2 fuzzy logic related to power gradient $m$ and its changes $\Delta m$ as input and duty ratio $\Delta D$ as crisp output.

Fig. 12 displays the transient response of the output power with a resistive load of $10 \mathrm{Ohm}$. The result appears that each method at steady state shows a good MPP value, but the transient process and steady state ripple are different. At interval 0-0.5, the T1FL showed a slightly faster response but had a less stable ripple than QL and QLT2FL which relatively smooth. In the 0.5-1 range, QL and QLT2FL showed a faster and ripples response. For interval 1-1.5, QL showed a fairly high undershoot, while QLT2FL yielded a significant improvement from the T2FL correction signal so that the response was faster, without ripple and reaches MPP more precisely. The value of the average output power efficiency against the maximum power at each interval is shown in 
Table 4 where the efficiency of QLT2FL is superior, which is $98.64 \%$.

Fig. 13 shows the transient response of the output power with a resistive load $50 \mathrm{Ohm}$. Across all ranges, the QLT2FL showed superior, faster, and smoother

Table 4. Power Tracking Efficiency

\begin{tabular}{|c|c|c|c|c|c|c|c|c|c|c|c|c|}
\hline \multirow{2}{*}{$\begin{array}{c}\text { Load } \\
\text { Value } \\
(\Omega)\end{array}$} & \multirow[b]{2}{*}{ Interval } & \multirow[b]{2}{*}{$\begin{array}{c}\text { MPP } \\
\text { (W) }\end{array}$} & \multicolumn{5}{|c|}{ Tracked Power $(\mathrm{W})$} & \multicolumn{5}{|c|}{ Tracking efficiency (\%) } \\
\hline & & & QLT2FL & QL & $\begin{array}{c}\text { T1FL- } \\
\text { triangle }\end{array}$ & \begin{tabular}{|c|} 
T1FL- \\
trapezoid
\end{tabular} & $\begin{array}{l}\text { T1FL- } \\
\text { gauss }\end{array}$ & QLT2FL & QL & $\begin{array}{l}\text { T1FL- } \\
\text { triangle }\end{array}$ & \begin{tabular}{|c|} 
T1FL- \\
trapezoid
\end{tabular} & $\begin{array}{l}\text { T1FL- } \\
\text { gauss }\end{array}$ \\
\hline \multirow{3}{*}{10} & $0-0.5$ & .15 & 12 & .08 & 80.60 & 8066 & 80.77 & 3.75 & 98.70 & 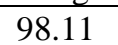 & 98.12 & 98.32 \\
\hline & 0.5 & & & 99 & 96.61 & 96.4 & 96.86 & 90 & 98.86 & 8. & 8.36 & 3.73 \\
\hline & & & & 9.88 & 41 & 89 . & 89.58 & 26 & 98.23 & 97. & .79 & 97.90 \\
\hline \multirow[t]{3}{*}{50} & $0-0.5$ & $s_{2}$ & & 79.73 & 77 & & 9 & 07 & 97.06 & & 6.09 & 95.91 \\
\hline & $0.5-1$ & & & 57 & & & 94.83 & & 97.52 & & .66 & 6.67 \\
\hline & & & & 52 & & & & & 96.74 & & .97 & 95.93 \\
\hline \multirow[t]{3}{*}{100} & & & & 94 & & & & & 93.66 & & 1.68 & 90.31 \\
\hline & & & & 4.30 & & & & 6.20 & 96.12 & & 4.60 & 94.84 \\
\hline & $1-1.5$ & 91.5 & 87.53 & 87.51 & 85.89 & 86.15 & 85.41 & 95.66 & 95.64 & 93.87 & 94.15 & 93.34 \\
\hline
\end{tabular}

response. However, in interval 1-1.5, QL showed a fairly high undershoot, contrast to QLT2FL which received a T2FL correction signal so that the response is faster, without ripple and more precise MPP. The value of the average output power efficiency against the maximum power at each interval is shown in Table 4 with load value $50 \mathrm{Ohm}$ where the efficiency of QLT2FL was leading around $97.12 \%$.

Fig. 14 shows the transient response of the output power with a resistive load $100 \mathrm{Ohm}$. In all ranges, the QLT2FL again showed superior response, faster and smoother. Unfortunately, interval 0.5-1, QL has undershoot, in contrast to QLT2FL which received a correction signal from the T2FL so that the response is faster, without ripple and reaches MPP more precisely. The value of the average output power efficiency against the maximum power at each interval is shown in Table 4 with load value $100 \mathrm{Ohm}$ where the QLT2FL efficiency is superior, which is $95.13 \%$.

The Fig. 15 (a) displays the system response from the photovoltaic module side. The top of figure is a system test scenario under varying of solar irradiation conditions and temperatures. The solar irradiation exposure value is divided into three intervals; 800 $\mathrm{W} / \mathrm{m}^{2}, 1000 \mathrm{~W} / \mathrm{m}^{2}$ and $900 \mathrm{~W} / \mathrm{m}^{2}$. For each irradiation interval, the temperature changes also occur, namely $25^{\circ} \mathrm{C}, 32^{\circ} \mathrm{C}$, and $27^{\circ} \mathrm{C}$. The solar energy that enters the photovoltaic was converted into electrical energy with a voltage simulation value $V_{P V}$, current $I_{P V}$ and photovoltaic power $P_{P V}$ as shown in middle and lower part of figure.

Fig. 15 (b) shows the response of the controller and electrical power generated by the system. At the top, two control signals are displayed, namely QL and T2FL, at time interval of 0-0.5 the incoming solar irradiation was $800 \mathrm{~W} / \mathrm{m}^{2}$ and photovoltaic voltage increases greater than $V_{m p p}$, consequently the controller, both from QL and T2FL, gradually reduce the value of the duty cycle. Consequently, this action caused the power gradient $m$ or $d P / d V$ moves from negative to zero, which means that the tracking point at right side of the MPP move to the left towards MPP until convergent. Moreover, the figure shows the comparative ability of fully automatic control mode and partial mode. The partial mode demonstrates power output while the condition is manually regulated which duty cycle sets constant by 0.5 , after that the regulation is changed manually to automatic transfer which the duty cycle applied from QLT2FL control. The result shows that the proposed Qlearning hybrid type-2 fuzzy logic control rise to achieve forward MPP successfully.

At 0.5 seconds, there is a gradually change in solar irradiation from $800 \mathrm{~W} / \mathrm{m}^{2}$ to $1000 \mathrm{~W} / \mathrm{m}^{2}$ causing $I_{P V}$ increase while $V_{P V}$ falls lower than the new $V_{m p p}$, so QL controller responds by step-up or increasing the duty-cycle. From the figure, it is observed that at the same time T2FL also adds a positive correction which results in the instantaneous positive forced power gradient or the power point moves to the left of the MPP and gradually converges back towards the new MPP.

After 1 second, solar irradiation changes from $1000 \mathrm{~W} / \mathrm{m}^{2}$ to $900 \mathrm{~W} / \mathrm{m}^{2}$ causing $\mathrm{I}_{\mathrm{Pv}}$ decrease 


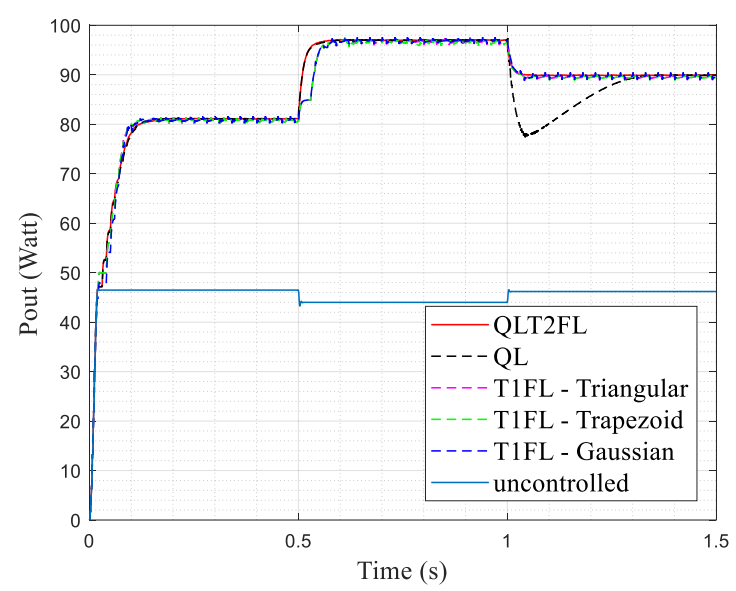

Figure. 12 Output power with resistive load $10 \Omega$

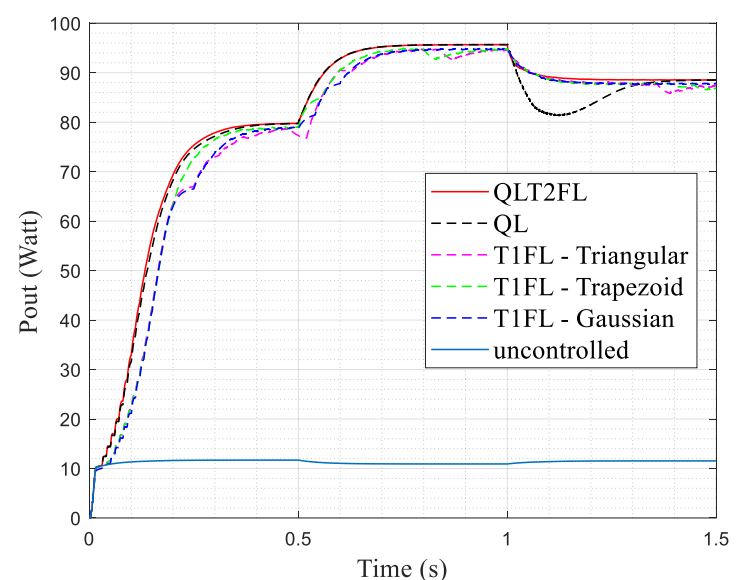

Figure. 13 Output power with resistive load $50 \Omega$

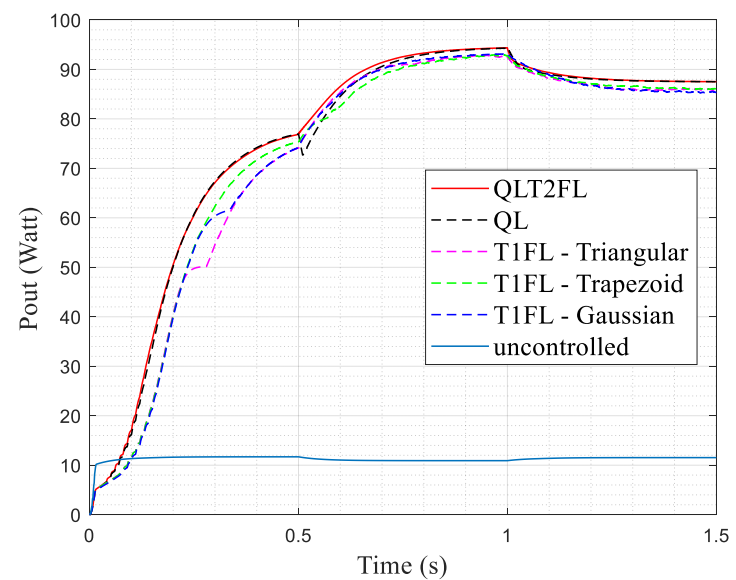

Figure. 14 Output power with resistive load $100 \Omega$

while $V_{P V}$ increases immediately higher than the new $V_{m p p}$. This situation was responded by the T2FL controller which gave a negative correction to $\mathrm{QL}$ resulted a temporary duty-ratio drop. Consequently, the power point moves oscillation around the MPP and then gradually converges back towards new MPP value.
In order to evaluate the performance of the QLT2FL strategy, the time responses of simulation result of power output shown in Figure. 12, 13 and 14 are summarize in Table 5. The response of the proposed strategy has no overshoot for each simulation test. The average rise time $\left(\mathrm{T}_{\mathrm{r}}\right)$ in resistive load evaluation is about $0.08 \mathrm{~s}$.

$\mathrm{T}_{\mathrm{r}}$ of QLT2FL is approximately 1.64 time faster than that of QL and 1.36 time faster than that of the average rise times of T1FL. Moreover, the average settling time $\left(\mathrm{T}_{\mathrm{s}}\right)$ of QLT2FL is approximately $0.23 \mathrm{~s}$ which means that is 1.6 time faster than that of the QL and T1FL.

Furthermore, the comparative study of performance evaluation of QLT2FL may
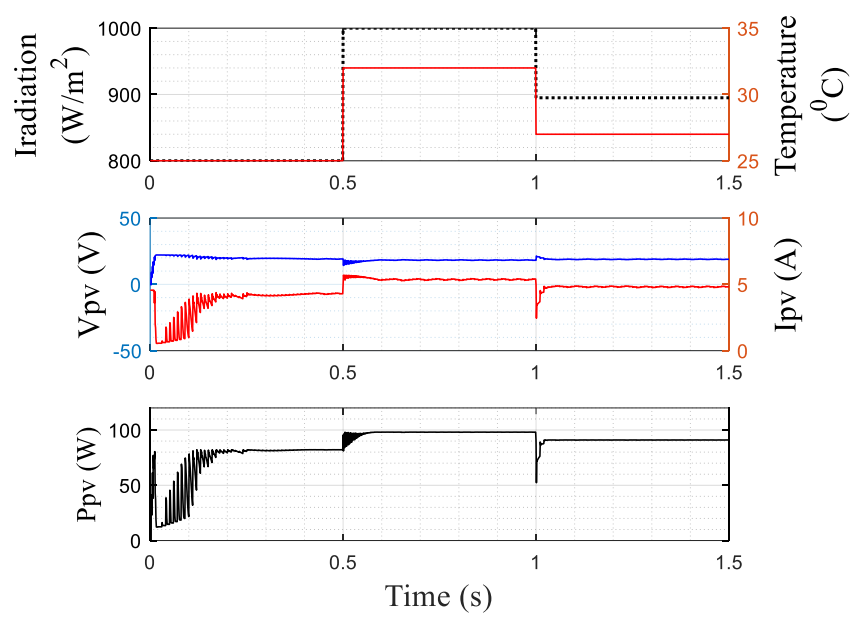

(a)
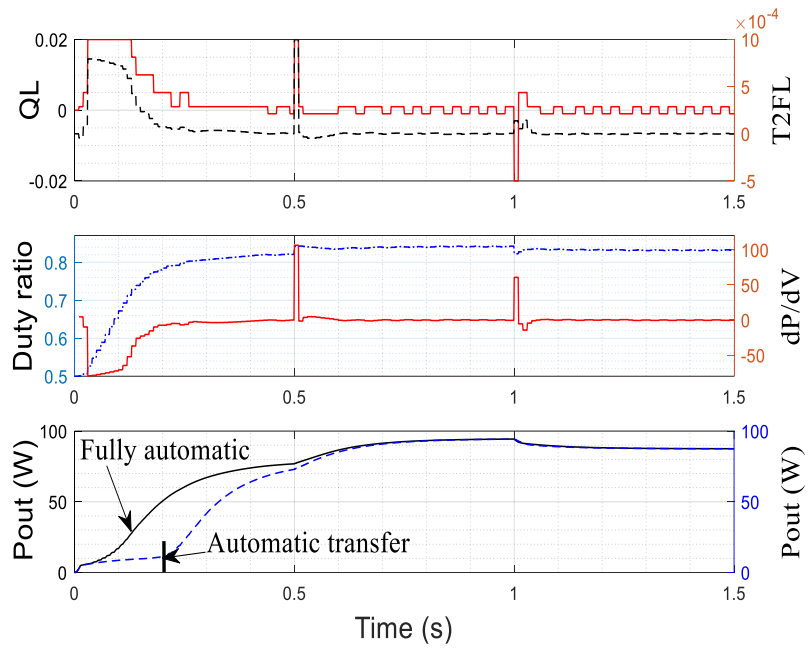

(b)

Figure. 15 System response: (a) Photovoltaic module response (b) controller response and power output with $\mathrm{R}_{\text {load }} 100 \Omega$

demonstrate with a well-known strategy under related condition, which is based hybrid fuzzy neural network (FNN) [10]. The FNN strategy performs tracking efficiency about $96 \%$, some of the 
simulation result in irradiation condition about 800 $\mathrm{W} / \mathrm{m}^{2}$ shows the overshoot due to temperature changes. As can be noted, the proposed method is more accurate which has tracking efficiency about 97\%. Moreover, QLT2FL is more stable response for handle the change of irradiation and temperature exposure simultaneously.

\section{Conclusion}

In this paper, Q-table hybrid with type-2 fuzzy logic control was proposed. A simple Q-tables trained to generate maximum conditions of possible states and actions gave the advantage in selecting the maximum action. The Q-learning hybrid method with

Table 5. Time response

\begin{tabular}{|c|c|c|c|c|c|c|c|c|c|c|}
\hline \multirow{2}{*}{\multicolumn{2}{|c|}{\begin{tabular}{|l|} 
Resistive Load \\
Interval
\end{tabular}}} & \multicolumn{3}{|c|}{$10 \Omega$} & \multicolumn{3}{|c|}{$50 \Omega$} & \multicolumn{3}{|c|}{$100 \Omega$} \\
\hline & & $0-0.5$ & $0.5-1$ & $1-1.5$ & $0-0.5$ & $0.5-1$ & $1-1.5$ & $0-0.5$ & $0.5-1$ & $1-1.5$ \\
\hline \multirow{5}{*}{$\begin{array}{l}\mathrm{T}_{\mathrm{r}} \\
(\mathrm{s})\end{array}$} & QLT2FL & 0.052 & 0.01 & 0.01 & 0.163 & 0.06 & 0.02 & 0.253 & 0.12 & 0.08 \\
\hline & $\mathrm{QL}$ & 0.542 & 0.01 & 0.006 & 0.17 & 0.06 & 0.015 & 0.253 & 0.13 & 0.06 \\
\hline & T1FL-triangle & 0.06 & 0.049 & 0.01 & 0.2 & 0.09 & 0.035 & 0.35 & 0.13 & 0.08 \\
\hline & T1FL-trapezoid & 0.06 & 0.049 & 0.01 & 0.2 & 0.09 & 0.03 & 0.3 & 0.16 & 0.08 \\
\hline & T1FL-gauss & 0.061 & 0.048 & 0.01 & 0.2 & 0.12 & 0.03 & 0.35 & 0.13 & 0.14 \\
\hline \multirow{5}{*}{$\begin{array}{l}\mathrm{T}_{\mathrm{s}} \\
(\mathrm{s})\end{array}$} & QLT2FL & 0.13 & 0.07 & 0.03 & 0.325 & 0.2 & 0.15 & 0.455 & 0.35 & 0.34 \\
\hline & QL & 0.14 & 0.08 & 0.34 & 0.325 & 0.2 & 0.45 & 0.455 & 0.955 & 0.34 \\
\hline & T1FL-triangle & 0.13 & 0.12 & 0.07 & 0.4 & 0.45 & 0.48 & 0.5 & 0.955 & 0.42 \\
\hline & T1FL-trapezoid & 0.13 & 0.12 & 0.07 & 0.325 & 0.45 & 0.48 & 0.5 & 0.955 & 0.42 \\
\hline & T1FL-gauss & 0.13 & 0.12 & 0.07 & 0.4 & 0.3 & 0.3 & 0.5 & 0.955 & 0.46 \\
\hline$\% \mathrm{OS}$ & QLT2FL & 0 & 0 & 0 & 0 & 0 & 0 & 0 & 0 & 0 \\
\hline
\end{tabular}

T2FL was proposed to provide correction of QL control signal by considering the direction uncertainty of power point shifting. The strategy was necessary particularly to avoid a sudden change in solar irradiation exposure. The simulation results showed that the QLT2FL-based MPPT response in the photovoltaic control system has faster rise time of about $0.08 \mathrm{~s}$ than that of the varying membership function of T1FL performance and stand-alone QL method, with settling time of $0.23 \mathrm{~s}$, low power oscillation, and with the average effective power tracking efficiency of about $97 \%$.

\section{Conflicts of interest}

The authors declare no conflict of interest.

\section{Author contributions}

Edi Leksono is corresponding author of this paper. $\mathrm{He}$ has supervised the writing of this paper and formal analysis of MPPT system performance. Endra Joelianto has contributed to the control strategy, he also reviews and edits the paper. Reza Fauzi Iskandar has designed the proposed control methodology, modeled the MPPT system in MATLAB/SIMULINK environment and writing the paper. All author approved the final version.

\section{Acknowledgments}

We sincerely thank to LPDP, Ministry of Finance, Republic of Indonesia for funding this research through Indonesian Education Scholarship program.

\section{References}

[1] M. Mao, L. Cui, Q. Zhang, K. Guo, L. Zhou, and H. Huang, "Classification and summarization of solar photovoltaic MPPT techniques: A review based on traditional and intelligent control strategies", Energy Reports, Vol. 6, pp. 13121327, 2020.

[2] S. Ma, M. Chen, J. Wu, W. Huo, and L. Huang, "Augmented nonlinear controller for maximum power-point tracking with artificial neural network in grid-connected photovoltaic systems", Energies, Vol. 9, No. 12, pp. 1-24, 2016.

[3] H. A. M. Kazim, I. A. Qader, and A. M. Harb, "Efficient maximum power point tracking based on reweighted zero-attracting variable stepsize for grid interfaced photovoltaic systems", Comput. Electr. Eng., Vol. 85, pp. 1-17, 2020.

[4] A. A. Kamil, M. S. Nasr, and S. Alwash, "Maximum Power Point Tracking Method for Photovoltaic System Based on Enhanced Particle Swarm Optimization Algorithm Under Partial Shading Condition", Int. J. Intell. Eng. Syst., Vol. 13, No. 6, pp. 241-254, 2020. 
[5] C. C. Ahmed, M. Cherkaoui, and M. Mokhlis, "PSO-SMC Controller Based GMPPT Technique for Photovoltaic Panel Under Partial Shading Effect", Int. J. Intell. Eng. Syst., Vol. 13, No. 2, pp. 307-316, 2020.

[6] K. Boudaraia, H. Mahmoudi, and A. Abbou, "MPPT Design Using Artificial Neural Network and Backstepping Sliding Mode Approach for Photovoltaic System under Various Weather Conditions", Int. J. Intell. Eng. Syst., Vol. 12, No. 6, pp. 177-186, 2019.

[7] M. Mokhlis, M. Ferfra, and R. E. Idrissi, "High Gain Observer-Based Control for GridConnected PV System Under Partial Shading Effect", Int. J. Intell. Eng. Syst., Vol. 13, No. 2, pp. 161-172, 2020.

[8] R. E. Idrissi, A. Abbou, and M. Mokhlis, "Backstepping Integral Sliding Mode Control Method for Maximum Power Point Tracking for Optimization of PV System Operation Based on High-Gain Observer", Int. J. Intell. Eng. Syst., Vol. 13, No. 5, pp. 133-144, 2020.

[9] D. Canny and F. Yusivar, "Maximum Power Point Tracking (MPPT) Algorithm Simulation Based on Fuzzy Logic Controller on Solar Cell with Boost Converter", In: 2nd Int. Conf. Smart Grid Smart Cities, Kuala Lumpur, Malaysia, pp. 117-121, 2018.

[10] W. I. Hameed, A. L. Saleh, B. A. Sawadi, Y. I. A. A. Yasir, and R. A. A. alhameed, "Maximum Power Point Tracking for Photovoltaic System by Using Fuzzy Neural Network", Inventions, Vol. 4, No. 3, pp. 1-12, 2019.

[11] O. Castillo, L. A. Angulo, J. R. Castro, and M. G. Aldez, "A comparative study of type-1 fuzzy logic systems, interval type-2 fuzzy logic systems and generalized type-2 fuzzy logic systems in control problems", Inf. Sci. (Ny)., Vol. 354, pp. 257-274, 2016.

[12] A. Orooji, M. Langarizadeh, M. Hassanzad, and M. R. Zarkesh, "A Comparison Between Fuzzy Type-1 and Type-2 Systems in Medical Decision Making: A Systematic Review", Crescent J. Med. Biol. Sci., Vol. 6, No. 3, pp. 246-252, 2019.

[13] T. Quah, D. Machalek, and K. M. Powell, "Comparing reinforcement learning methods for real-time optimization of a chemical process", Processes, Vol. 8, No. 11, pp. 1-19, 2020.

[14] C. Deng, X. Ji, C. Rainey, J. Zhang, and W. Lu, "Integrating Machine Learning with Human Knowledge", iScience, Vol. 23, No. 11. pp. 127, Nov. 20, 2020.

[15] Y. Chaibi, A. Allouhi, M. Malvoni, M. Salhi, and R. Saadani, "Solar irradiance and temperature influence on the photovoltaic cell equivalent-circuit models", Sol. Energy, Vol. 188, pp. 1102-1110, 2019.

[16] M. E. Şahin and H. İ. Okumuş, "Comparison of Different Controllers and Stability Analysis for Photovoltaic Powered Buck-Boost DC-DC Converter", Electr. Power Components Syst., Vol. 46, No. 2, pp. 149-161, 2018.

[17] K. Y. Chou, S. T. Yang, and Y. P. Chen, "Maximum power point tracking of photovoltaic system based on reinforcement learning", Sensors, Vol. 19, No. 22, pp. 1-22, 2019.

[18] N. Bruchon, G. Fenu, G. Gaio, M. Lonza, F. H. O. Shea, F. A. Pellegrino, and E. Salvato, "Basic Reinforcement Learning Techniques to Control the Intensity of a Seeded Free-Electron Laser", Electronics, Vol. 9, No. 5, pp. 1-15, 2020.

[19] K. Mittal, A. Jain, K. S. Vaisla, O. Castillo, and J. Kacprzyk, "A comprehensive review on type 2 fuzzy logic applications: Past, present and future", Eng. Appl. Artif. Intell., Vol. 95, pp. 112,2020

[20] A. Taskin and T. Kumbasar, "An open source matlab/simulink toolbox for interval type-2 fuzzy logic systems", In: Proc. - 2015 IEEE Symp. Ser. Comput. Intell, Cape Town, South Africa, pp. 1561-1568, 2015. 